\title{
Influence of False Lumen Status on the Prognosis of Acute Type A Aortic Dissection without Urgent Surgical Treatment
}

\author{
Akihito Tanaka ${ }^{1}$, Hideki Ishii ${ }^{1}$, Susumu Suzuki ${ }^{1}$, Tomoyuki Ota ${ }^{1}$, Hideki Oshima ${ }^{2}$, Akihiko Usui ${ }^{2}$, \\ Kimihiro Komori ${ }^{3}$ and Toyoaki Murohara ${ }^{1}$ \\ ${ }^{1}$ Department of Cardiology, Nagoya University Graduate School of Medicine, Nagoya, Japan \\ ${ }^{2}$ Department of Cardiac Surgery, Nagoya University Graduate School of Medicine, Nagoya, Japan \\ ${ }^{3}$ Department of Vascular Surgery, Nagoya University Graduate School of Medicine, Nagoya, Japan
}

\begin{abstract}
Aim: Recently, much attention has been focused on partial thrombosis of the false lumen in patients with acute aortic dissection. However, its effect on clinical outcomes in these patients, especially in case of acute type A aortic dissection, has not been clearly elucidated. This study evaluated the influence of the false lumen status, including partial thrombosis, on short-term clinical outcomes in acute type A aortic dissection patients without urgent surgical treatment.

Methods: Sixty-two patients ( 29 males, mean age $73 \pm 13$ years) with acute type A aortic dissection who did not receive urgent surgical treatment at four hospitals were enrolled. Patients were divided into three groups based on the false lumen status on enhanced computed tomography image (complete thrombosis, $n=28$; partial thrombosis, $n=27$; patent, $n=7$ ). Patients with partial thrombosis were further divided into two groups (thrombus-dominant, $n=15$; flow-dominant, $n=12$ ).

Results: The short-term mortality rate (in-hospital and 30-day) was significantly higher in patients with a patent false lumen, while no significant difference was seen between the other two groups. Patients with flow-dominant partial thrombosis had significantly higher short-term mortality rate than those with thrombus-dominant partial thrombosis (in-hospital, $p=0.001$ and 30 -day, $p<0.001$ ). Conclusions: The short-term mortality rate in acute type A aortic dissection patients without urgent surgical treatment was lower in patients with partial thrombosis of the false lumen than in those with a patent false lumen. Furthermore, patients with flow-dominant partial thrombosis had higher mortality rate than those with thrombus-dominant partial thrombosis.
\end{abstract}

Key words: Type A aortic dissection, False lumen status, Partial thrombosis

This article is distributed under the terms of the latest version of CC BY-NC-SA defined by the Creative Commons Attribution License.

\section{Introduction}

A recent report showed that partial thrombosis of the false lumen is an independent predictor of longterm mortality in patients with acute type B aortic dissection ${ }^{1)}$. Thereafter, much attention has been focused on partial thrombosis of the false lumen in patients with acute aortic dissection ${ }^{2-8)}$. However, the issue about partial thrombosis of the false lumen has not been clearly elucidated. Furthermore, the extent of partial thrombosis has not been fully studied in

Address for correspondence: Akihito Tanaka, Department of Cardiology, Nagoya University Graduate School of Medicine,

65 Tsurumai-cho, Showa-ku, Nagoya, 466-8550, Japan

E-mail: akihito17491194@gmail.com

Received: March 13, 2016

Accepted for publication: June 26, 2016 these patients ${ }^{6}$.

Type A dissection is associated with higher mortality, and often involves hemodynamic instability ${ }^{9-11)}$. Therefore, research focused on type A dissection may contribute towards improving our understanding of the significance of false lumen status in patients with acute aortic dissection. However, urgent surgical repair is generally indicated for type A dissection ${ }^{9}$, and data regarding false lumen status in type A dissection patients who do not undergo urgent surgical treatment are scarce.

\section{Aim}

The aim of this study was to evaluate the influence of the false lumen status, including partial thrombosis, on short-term clinical outcomes in acute 

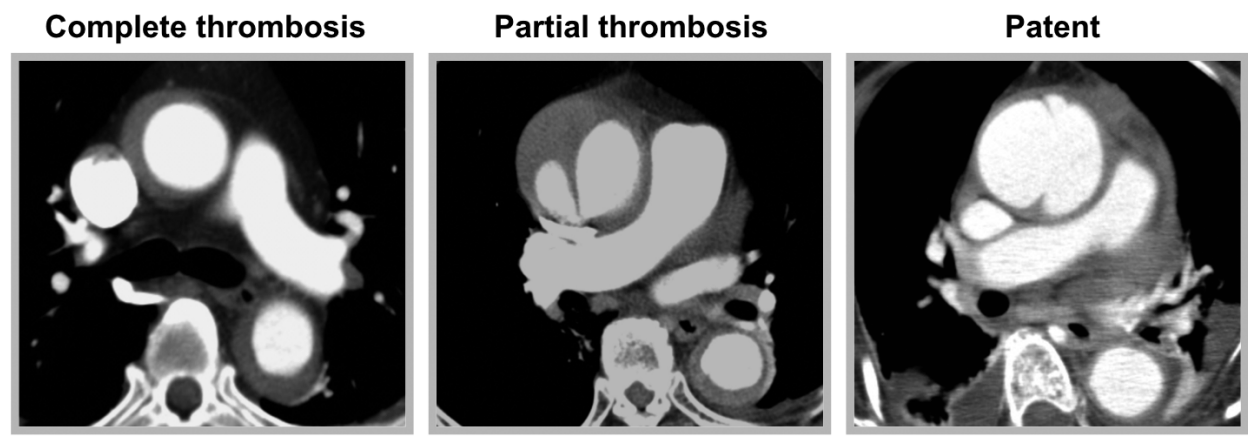

Fig. 1. Enhanced computed tomography images, which show complete thrombosis, partial thrombosis, and patent false lumen

type A aortic dissection patients without urgent surgical treatment.

\section{Methods}

Data of acute type A aortic dissection patients who were admitted and received initial medical treatment in four hospitals (Nagoya University Hospital, Yokkaichi Municipal Hospital, Toyota Memorial Hospital, and Handa City Hospital) between 2004 and 2013 were examined $(n=62)$. Acute type A aortic dissection was defined as any non-traumatic dissection involving the ascending aorta and presenting within 14 days of the onset of symptoms. The diagnosis was confirmed by enhanced computed tomography (CT) immediately after emergency admission. Urgent surgical treatment was generally considered for all patients, and the final decision was based on various factors, including each patient's comorbidities and general condition. Initial medical treatment was defined as treatment not involving any surgical intervention during the first four days of admission. Blood pressure was controlled to maintain systolic blood pressure below $120 \mathrm{~mm} \mathrm{Hg}$ immediately after admission, using continuous intravenous infusion and oral administration of antihypertensive drugs ${ }^{12)}$.

This study was performed according to the guidelines of the Declaration of Helsinki and approved by the local Ethics Committee, which waived the requirement for written informed consent.

Imaging results were interpreted at each patient's hospital by experienced cardiologists and radiologists. On imaging, the status of the false lumen was classified as completely thrombosed if no flow was present, as partially thrombosed if both flow and thrombus were present, and as patent if flow was present in the absence of thrombus. Thus, all the patients were divided into three groups based on the status of the false lumen on initial CT image: patent, partial thrombosis, and complete thrombosis (Fig. 1). Furthermore, patients with partial thrombosis of the false lumen were divided into two groups, thrombus-dominant partial thrombosis if more than $50 \%$ of the false lumen was thrombosed of the total length of dissected aorta on visual inspection, and flow-dominant partial thrombosis in the remaining cases.

All patients were assessed for the occurrence of short-term clinical events, including all-cause death and late surgical treatment related to aortic dissection.

Data are presented as mean \pm standard deviation. Categorical variables are expressed as count and percentages. Categorical data were compared using the $\chi^{2}$ test or Fisher exact test. Continuous data were compared using an unpaired $t$ test. Among the three groups, continuous data were compared using analysis of variance. Kaplan-Meier estimates were generated to describe outcome time-course. The log-rank test was used to compare differences. A $p$-value of $<0.05$ was considered statistically significant. All analyses were performed with the SPSS 18.0 software package (SPSS, Chicago, IL, USA).

\section{Results}

The mean age of the enrolled patients was $73 \pm$ 13 years (Table 1). Majority of these patients $(83.9 \%)$ had history of hypertension; only $1.6 \%$ had diabetes. Previous aortic dissection (12.9\%) and aortic aneurysm (19.4\%) were not uncommon.

Table 1 shows baseline characteristics of patients stratified according to the status of the false lumen, and major reasons for the initial medical treatment. On CT imaging, the false lumen was found to be completely thrombosed in 28 patients (45.2\%), partially thrombosed in $27(43.5 \%)$, and patent in 7 $(11.3 \%)$. There were no significant differences in age, sex, clinical characteristics, and clinical presentations among the three groups. 
Table 1. Patient characteristics

\begin{tabular}{|c|c|c|c|c|c|}
\hline & $\begin{array}{c}\text { All } \\
n=62\end{array}$ & $\begin{array}{c}\text { Complete } \\
n=28\end{array}$ & $\begin{array}{l}\text { Partial } \\
n=27\end{array}$ & $\begin{array}{c}\text { Patent } \\
n=7\end{array}$ & $P$-value \\
\hline \multicolumn{6}{|l|}{ Characteristic } \\
\hline Age, years & $73 \pm 13$ & $72 \pm 11$ & $72 \pm 15$ & $78 \pm 8$ & 0.49 \\
\hline Men & $29(46.8 \%)$ & $11(39.3 \%)$ & $15(55.6 \%)$ & $3(42.9 \%)$ & 0.47 \\
\hline Hypertension & $52(83.9 \%)$ & $27(96.4 \%)$ & $20(74.1 \%)$ & $5(71.4 \%)$ & 0.05 \\
\hline Dyslipidemia & $16(25.8 \%)$ & $8(28.6 \%)$ & $8(29.6 \%)$ & $0(0 \%)$ & 0.25 \\
\hline Diabetes & $1(1.6 \%)$ & $0(0 \%)$ & $0(0 \%)$ & $1(14.3 \%)$ & 0.02 \\
\hline Current smoker & $13(21 \%)$ & $4(14.3 \%)$ & $8(29.6 \%)$ & $1(14.3 \%)$ & 0.34 \\
\hline Hemodialysis & $2(3.2 \%)$ & $1(3.6 \%)$ & $1(3.7 \%)$ & $0(0 \%)$ & 0.88 \\
\hline Marfan syndrome & $2(3.2 \%)$ & $0(0 \%)$ & $2(7.4 \%)$ & $0(0 \%)$ & 0.26 \\
\hline Previous aortic dissection & $8(12.9 \%)$ & $2(7.1 \%)$ & $4(14.8 \%)$ & $2(28.6 \%)$ & 0.30 \\
\hline Previous aortic aneurysm & $12(19.4 \%)$ & $4(14.3 \%)$ & $8(29.6 \%)$ & $0(0 \%)$ & 0.14 \\
\hline Previous cardiac surgery & $4(6.5 \%)$ & $0(0 \%)$ & $4(14.8 \%)$ & $0(0 \%)$ & 0.06 \\
\hline \multicolumn{6}{|l|}{ Clinical presentation } \\
\hline Chest/back pain & $53(85.5 \%)$ & $26(92.9 \%)$ & $21(77.8 \%)$ & $6(85.7 \%)$ & 0.28 \\
\hline Abrupt onset of pain & $45(72.6 \%)$ & $22(78.6 \%)$ & $20(74.1 \%)$ & $3(42.9 \%)$ & 0.16 \\
\hline Migration of pain & $8(12.9 \%)$ & $3(10.7 \%)$ & $5(18.5 \%)$ & $0(0 \%)$ & 0.38 \\
\hline Hypotension & $17(27.4 \%)$ & $7(25.0 \%)$ & $7(25.9 \%)$ & $3(42.9 \%)$ & 0.62 \\
\hline Any neurologic deficit & $15(24.2 \%)$ & $4(14.3 \%)$ & $8(29.6 \%)$ & $3(42.9 \%)$ & 0.20 \\
\hline Aortic valve regurgitation & $15(24.2 \%)$ & $6(21.4 \%)$ & $6(22.2 \%)$ & $3(42.9 \%)$ & 0.47 \\
\hline Widest diameter of ascending aorta, $\mathrm{mm}$ & $49.0 \pm 8.1$ & $48.3 \pm 8.7$ & $48.4 \pm 7.7$ & $53.8 \pm 6.3$ & 0.25 \\
\hline Pericardial effusion & $29(46.8 \%)$ & $13(46.4 \%)$ & $12(44.4)$ & $4(57.1 \%)$ & 0.83 \\
\hline \multicolumn{6}{|l|}{ Reason for nonoperative management } \\
\hline Refusal of operation (dementia, frailty, high age) & $23(37.1 \%)$ & $8(28.6 \%)$ & $12(44.4 \%)$ & $3(42.9 \%)$ & \\
\hline Delay in diagnosis & $3(4.8 \%)$ & $1(3.6 \%)$ & $0(0 \%)$ & $2(28.6 \%)$ & \\
\hline Extensive comorbidity & $8(12.9 \%)$ & $0(0 \%)$ & $6(22.2 \%)$ & $2(28.6 \%)$ & \\
\hline Encephalopathy & & & 2 & 2 & \\
\hline Advanced cancer & & & 1 & 0 & \\
\hline Prior thoracic surgery & & & 3 & 0 & \\
\hline Thrombosis of the false lumen in ascending aorta & $25(40.3 \%)$ & $18(64.3 \%)$ & $7(25.9 \%)$ & $0(0 \%)$ & \\
\hline Other & $3(4.8 \%)$ & $1(3.6 \%)$ & $2(7.4 \%)$ & $0(0 \%)$ & \\
\hline
\end{tabular}

Table 2 shows baseline characteristics of patients with thrombus-dominant partial thrombosis and flowdominant partial thrombosis. The patients with thrombus-dominant partial thrombosis tended to be older with higher rates of aortic regurgitation and pericardial effusion; however, no statistically significant differences were seen.

Fig. 2 shows Kaplan-Meier survival curves indicating freedom from all-cause death (2A), and allcause death and late surgical treatment (2B) in patients stratified according to the status of the false lumen and compares the three groups (complete thrombosis, partial thrombosis, and patent). Table 3 shows details of the in-hospital outcomes. Event-free rates were lowest in patients with a patent false lumen, while no significant differences were seen between patients with complete and partial thrombosis of the false lumen.
Fig. 3 shows Kaplan-Meier survival curves indicating freedom from all-cause death $(3 \mathrm{~A})$, and allcause death and late surgical treatment (3B) in patients with partial thrombosis of the false lumen and compares patients with flow-dominant partial thrombosis and thrombus-dominant partial thrombosis. Table 3 shows the detail of in-hospital outcomes. Patients with flow-dominant partial thrombosis had significantly lower event-free rates than those with thrombus-dominant partial thrombosis.

\section{Discussion}

This study showed that the short-term mortality rate in acute type $\mathrm{A}$ aortic dissection patients without urgent surgical treatment was lower in patients with partial thrombosis of the false lumen than in those with a patent false lumen. Furthermore, in case of 
Table 2. Patients characteristics (thrombus partial vs. flow partial)

\begin{tabular}{lccc}
\hline & Thrombus dominant partial & Flow dominant partial & \\
& $n=15$ & $n=12$ & $P$-value \\
\hline Age, y & $75 \pm 14$ & $69 \pm 16$ & 0.33 \\
Men & $8(53.3 \%)$ & $7(58.3 \%)$ & 0.80 \\
Hypertension & $11(73.3 \%)$ & $9(75.0 \%)$ & 1.00 \\
Dyslipidemia & $7(46.7 \%)$ & $1(8.3 \%)$ & 0.04 \\
Diabetes & $0(0 \%)$ & $0(0 \%)$ & \\
Current smoker & $6(40.0 \%)$ & $2(16.7 \%)$ & 0.24 \\
& & & \\
Aortic valve regurgitation & $5(33.3 \%)$ & $1(8.3 \%)$ & 0.18 \\
Widest diameter of ascending aorta, mm & $48.8 \pm 8.6$ & $47.9 \pm 6.8$ & 0.77 \\
Pericardial effusion & $8(53.3 \%)$ & & 0.44 \\
& & & \\
Ends filled with thrombus in false lumen & & $3(25 \%)$ & \\
$\quad$ Both ends & $14(93.3 \%)$ & $6(50 \%)$ & \\
$\quad$ Only proximal end & $1(6.7 \%)$ & $3(25 \%)$ & \\
Only distal end & $0(0 \%)$ & & \\
\hline
\end{tabular}
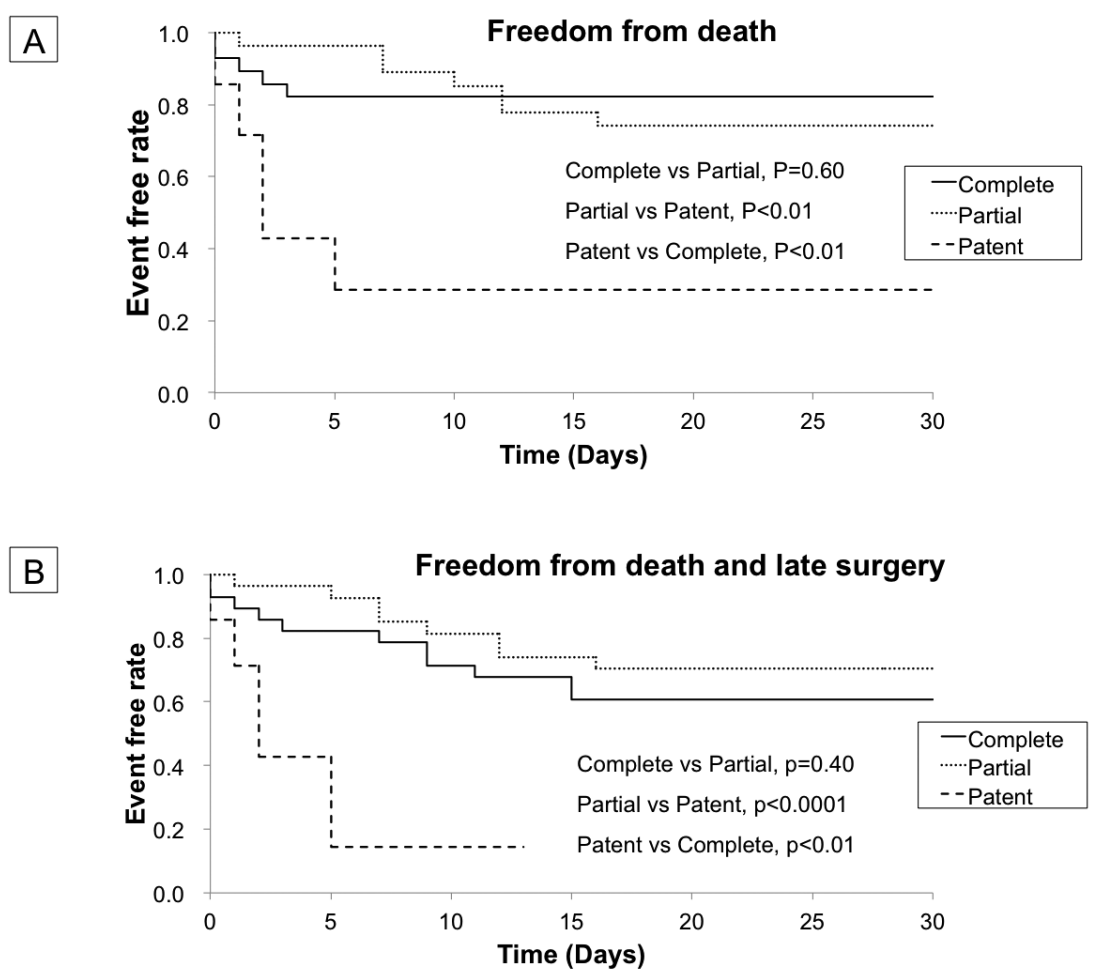

Fig. 2A. Kaplan-Meier survival curves stratified according to false lumen status (complete, partial, and patent)

The event free rate was higher in patients with partial thrombosis than patent false lumen. There was no significant difference between patients with partial thrombosis and complete thrombosis.

Fig. 2B. Kaplan-Meier survival curves indicating freedom from all cause death and late surgical treatment in all patients

The event free rate was higher in patients with partial thrombosis than patent false lumen. There was no significant difference between patients with partial thrombosis and complete thrombosis. 
Table 3. In hospital outcomes

\begin{tabular}{|c|c|c|c|c|c|c|c|}
\hline & \multirow[t]{2}{*}{ All } & \multirow[t]{2}{*}{ Complete } & \multicolumn{4}{|c|}{ Partial thrombosis } & \multirow[t]{2}{*}{ Patent } \\
\hline & & & $\begin{array}{l}\text { All Partial } \\
\qquad n=27\end{array}$ & $\begin{array}{l}\text { Thrombus- } \\
\text { dominant } \\
n=15\end{array}$ & $\begin{array}{c}\text { Flow- } \\
\text { dominant } \\
n=12\end{array}$ & $P$-value* & \\
\hline Death & $18(29 \%)$ & $6(21.4 \%)$ & $7(25.9 \%)$ & $0(0 \%)$ & $7(58.3 \%)$ & $0.001^{*}$ & $5(71.4 \%)$ \\
\hline Late surgical treatment & $11(17.7 \%)$ & $7(25 \%)$ & $3(11.1 \%)$ & $1(6.7 \%)$ & $2(16.7 \%)$ & $0.57^{*}$ & $1(14.3 \%)$ \\
\hline
\end{tabular}

*Thrombus-dominant partial thrombosis vs. Flow-dominant partial thrombosis

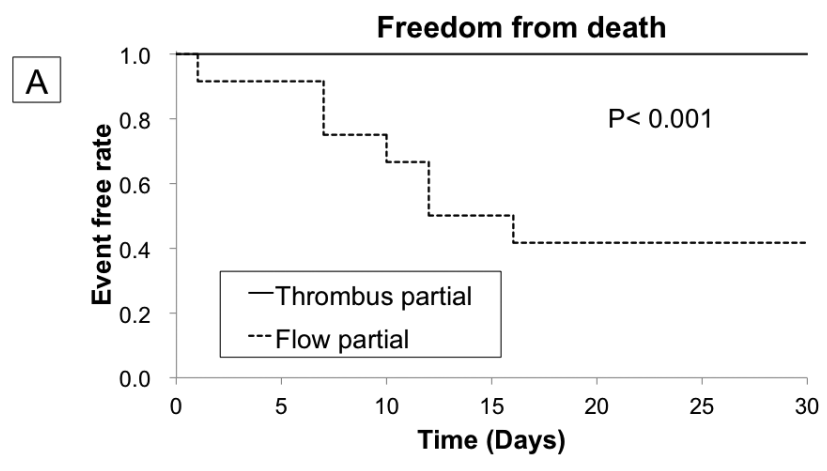

Freedom from death and late surgery

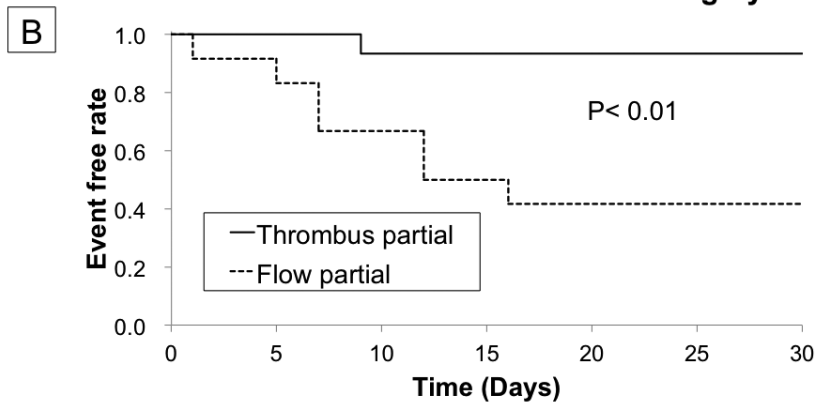

Fig. 3A. Kaplan-Meier survival curves in patients with partial thrombosis, and compared flow dominant partial thrombosis and thrombus dominant partial thrombosis

The event free rate was lower in patients with flow dominant partial thrombosis than thrombus dominant partial thrombosis.

Fig. 3B. Kaplan-Meier survival curves indicating freedom from all cause death and late surgical treatment in patients with partial thrombosis

The event free rate was lower in patients with flow dominant partial thrombosis than thrombus dominant partial thrombosis.

patients with partial thrombosis of the false lumen, those with flow-dominant partial thrombosis had higher mortality rate than those with thrombus-dominant partial thrombosis.

With regard to type B aortic dissection, some studies have shown that patients with partial thrombosis of the false lumen have higher mortality ${ }^{1)}$ and aortic growth rate ${ }^{2,7)}$ than those with a patent false lumen or complete thrombosis of the false lumen. On the other hand, another study demonstrated that the growth rate of the affected aorta among the three groups is highest in patients with a patent false lumen ${ }^{8)}$. We have previously reported that the longterm mortality does not significantly differ among the 
three groups ${ }^{6}$. Some reports refer to the partial thrombosis of the false lumen in type A aortic dissection. Larsen et al. showed that preoperative partial thrombosis of the false lumen in surgically treated type A aortic dissection is not an independent predictor of long-term outcomes ${ }^{3)}$. Other reports showed partial thrombosis of the residual false lumen after surgical repair to be a significant independent predictor of aortic enlargement ${ }^{4}$ and poor long-term survival $^{5)}$. Thus, at present, results regarding partial thrombosis of the false lumen are conflicting.

As previously reported ${ }^{6}$, it is important to study the extent of partial thrombosis in patients with acute aortic dissection. In this context, we consider the proportion of the thrombosed lumen to the false lumen as one of the main parameters. Although Tsai et al. mentioned that the formation of partial thrombosis may occlude distal tears and impede outflow ${ }^{1)}$, the effects of a $90 \%$ thrombosed false lumen might be different from those of a $10 \%$ thrombosed false lumen. However, previous studies could not reveal these differences in respect of partial thrombosis because of small sample sizes.

In this study, the patients with partial thrombosis of the false lumen did not have worse clinical outcomes than those with a patent false lumen. Furthermore, we delved into the issue of extent of partial thrombosis. Patients with partial thrombosis of the false lumen were divided into two groups based on the proportion of the thrombosed lumen to the false lumen. Patients with flow-dominant partial thrombosis had significantly higher short-term mortality rate than those with thrombus-dominant partial thrombosis. Although it may be a little premature, the results of this study suggest the extent of thrombosed false lumen can be used as risk stratification, and would lead to revealing of the diversity of partial thrombosis.

This study has a number of limitations. First, sample size was relatively small. Second, imaging techniques might have varied across the hospitals contributing to possible misclassification of the false lumen status. Third, the proportion of the thrombus in the false lumen was assessed only by visual inspection when classified into thrombus-dominant or flowdominant partial thrombosis. Fourth, non-operative management was decided by the attending team of each hospital over the study period, and the reasons were varied, which might bias the results. Fifth, this study involved only non-surgical candidates and we did not compare our results against those who underwent urgent surgery, and therefore cannot comment on whether initial medical treatment is a valid option. Sixth, our data did not contain the data of laboratory examinations including D-dimer. However, at present, there are only few studies on the status of the false lumen in patients with type A aortic dissection and especially the extent of partial thrombosis in these patients. Thus, it would be beneficial to accumulate data on this issue.

\section{Conclusion}

Patients with partial thrombosis of the false lumen did not have worse short-term clinical outcomes than those with complete thrombosis of the false lumen or a patent false lumen in case of type A aortic dissection patients without urgent surgical treatment. Furthermore, the extent of partial thrombosis might influence clinical outcomes in these patients.

\section{Acknowledgment}

The authors thank Dr. Satoshi Ichimiya, Dr. Masaaki Kanashiro and Dr. Junji Watanabe from Yokkaichi Municipal Hospital, Dr. Hisashi Umeda, Dr. Ryoji Ishiki and Dr. Mitsunori Iwase from Toyota Memorial Hospital, and Dr. Masaki Sakakibara from Handa City Hospital for their support of data collection.

\section{Conflict of Interest}

Dr. Ishii has received lecture fees from Astellas, Daiichi-Sankyo and Otsuka. Dr. Murohara has received lecture fees from Bayer, Daiichi Sankyo, Dainippon Sumitomo, Kowa, MSD, Mitsubishi Tanabe, Nippon Boehringer Ingelheim, Novartis, Pfizer Japan, Sanofi-Aventis, and Takeda. Dr. Usui has received lecture fees from Japan Blood Products Organization, and Terumo. Dr. Komori has received lecture fees from Sanofi-Aventis, Otuka, Daiichi Sankyo. Dr. Murohara has received unrestricted research grant for Department of Cardiology, Nagoya University Graduate School of Medicine, from Astellas, Daiichi Sankyo, Dainippon Sumitomo, Kowa, MSD, Mitsubishi Tanabe, Nippon Boehringer Ingelheim, Novartis, Otsuka, Pfizer Japan, Sanofi-Aventis, Takeda, and Teijin. Dr. Usui has receive unrestricted research grant for Department of Cardiac Surgery, Nagoya University Graduate School of Medicine, from Terumo, Medtronic, Edwards, Japan Lifeline, Senko Medical Instrument, and St. Jude Medical Japan. Dr. Komori has received unrestricted research grant for Department of Vascular Surgery, Nagoya University Graduate School of Medicine, from Sanofi-Aventis, Otsuka, Daiichi Sankyo, Eisai, and Gore Japan. 


\section{Funding}

This work was not supported by additional funding.

\section{Review}

This work was presented at ESC congress 2015.

\section{References}

1) Tsai TT, Evangelista A, Nienaber CA, Myrmel T, Meinhardt G, Cooper JV, Smith DE, Suzuki T, Fattori R, Llovet A, Froehlich J, Hutchison S, Distante A, Sundt T, Beckman J, Januzzi JL, Jr., Isselbacher EM, Eagle KA: Partial thrombosis of the false lumen in patients with acute type B aortic dissection. N Engl J Med, 2007; 357: 349-359

2) Tolenaar JL, Eagle KA, Jonker FH, Moll FL, Elefteriades JA, Trimarchi S: Partial thrombosis of the false lumen influences aortic growth in type B dissection. Ann Cardiothorac Surg, 2014; 3: 275-277

3) Larsen M, Bartnes K, Tsai TT, Eagle KA, Evangelista A, Nienaber CA, Suzuki T, Fattori R, Froehlich JB, Hutchison S, Sundt TM, Januzzi JL, Isselbacher EM, Montgomery DG, Myrmel T: Extent of preoperative false lumen thrombosis does not influence long-term survival in patients with acute type A aortic dissection. J Am Heart Assoc, 2013; 2: e000112

4) Tsai MT, Wu HY, Roan JN, Tsai YS, Hsieh PC, Yang YJ, Luo CY: Effect of false lumen partial thrombosis on repaired acute type A aortic dissection. J Thorac Cardiovasc Surg, 2014; 148: 2140-2146 e3

5) Song SW, Chang BC, Cho BK, Yi G, Youn YN, Lee S,
Yoo KJ: Effects of partial thrombosis on distal aorta after repair of acute DeBakey type I aortic dissection. J Thorac Cardiovasc Surg, 2010; 139: 841-847

6) Tanaka A, Sakakibara M, Ishii H, Hayashida R, Jinno Y, Okumura S, Okada K, Murohara T: Influence of the false lumen status on clinical outcomes in patients with acute type B aortic dissection. J Vasc Surg, 2014; 59: 321-326

7) Trimarchi S, Tolenaar JL, Jonker FH, Murray B, Tsai TT, Eagle KA, Rampoldi V, Verhagen HJ, van Herwaarden JA, Moll FL, Muhs BE, Elefteriades JA: Importance of false lumen thrombosis in type B aortic dissection prognosis. J Thorac Cardiovasc Surg, 2013; 145: S208-212

8) Sueyoshi E, Sakamoto I, Uetani M: Growth rate of affected aorta in patients with type B partially closed aortic dissection. Ann Thorac Surg, 2009; 88: 1251-1257

9) Bonaca MP, O'Gara PT: Diagnosis and management of acute aortic syndromes: dissection, intramural hematoma, and penetrating aortic ulcer. Curr Cardiol Rep, 2014; 16: 536

10) Hagan PG, Nienaber CA, Isselbacher EM, Bruckman D, Karavite DJ, Russman PL, Evangelista A, Fattori R, Suzuki T, Oh JK, Moore AG, Malouf JF, Pape LA, Gaca C, Sechtem U, Lenferink S, Deutsch HJ, Diedrichs H, Marcos y Robles J, Llovet A, Gilon D, Das SK, Armstrong WF, Deeb GM, Eagle KA: The International Registry of Acute Aortic Dissection (IRAD): new insights into an old disease. JAMA, 2000; 283: 897-903

11) Howard DP, Banerjee A, Fairhead JF, Perkins J, Silver LE, Rothwell PM: Population-based study of incidence and outcome of acute aortic dissection and premorbid risk factor control: 10-year results from the Oxford Vascular Study. Circulation, 2013; 127: 2031-2037

12) Guidelines for diagnosis and treatment of aortic aneurysm and aortic dissection (JCS 2011): digest version. Circ J, 2013; 77: 789-828 